\title{
Efficient Design of Dynamic Systems by Combining Network Methods with Finite-Element Methods
}

\author{
U. Marschner ${ }^{1}$, E. Starke ${ }^{2}$, G. Pfeifer ${ }^{1}$ \\ ${ }^{1}$ Institute for Semiconductors and Microsystems Technology, Technische Universität Dresden, \\ 01062 Dresden, Germany, uwe.marschner@tu-dresden.de \\ ${ }^{2}$ Institute of Lightweight Engineering and Polymer Technology, Holbeinstr. 3 \\ 01307 Dresden, Germany
}

\begin{abstract}
The predetermination of the dynamic behavior of electromechanical systems is a challenging task because of the coupled physical domains and various transducer principles. An efficient design of such systems requires simulation methods which permit a simultaneous design-accompanying optimization, too. The combination of network methods and Finite Element methods on user level is a timeefficient method for the simulation of the dynamic behavior of electromechanical systems. In this paper the advantages and the efficiency of this Combined Simulation are demonstrated by hand of different examples in the light of different abstraction levels. The examples include the application of FEsimulations to determine network structures and parameters, as well as the inclusion of network elements and equivalent network structures in FE-models.
\end{abstract}

Key words: System design, Finite Element method, Heterogeneous models, Multiphysics, System optimization, Equivalent Circuit

\section{Introduction}

The numerical optimization of the dynamic behavior of multi-domain systems requires models and techniques which are appropriate for a fast behavioral simulation. Today a variety of simulation software is available, which is intended for solving individual problems, like electromagnetic fields, modal analysis or circuit analysis. Applied methods include Finite-Element Methods (FEM), Boundary Element Methods (BEM) and involve hardware specification languages, like HDL, HDL-AMS, Verilog, and others. Distinguished methods by its efficiency are network methods [1] and signal-coupled networks without inherent feedback [2]. Depending on a concrete setting of tasks the use of an individual simulation method might lead to unsatisfactory results regarding computing time or accuracy which calls the system analysis with a single simulator into question. For this reason especially with very closely coupled effects two, maximum three simulators are coupled $[3,4]$.

A different approach is to combine different simulation methods [5]. A powerful combination is that of Finite-Element programs with network analysis tools, which are the most common simulation tools used in enterprises by design engineers. This combination allows the handling of complex systems by increasing the abstraction in a hierarchical model, which sim- plifies the behavioral simulation. For this reason network analysis programs have been integrated in widespread system simulation programs, such as ANSYS ${ }^{\circledR}$ or the Wolfram system modeler during the last years.

In the next sections different applications of Combined Simulation are demonstrated in the light of different abstraction levels.

\section{Hierarchical System Models}

Complex multi-domain systems are commonly modeled by introducing a hierarchy. A system is typically partitioned into the four levels shown in Fig. 1. The lower levels comprise more detail than the higher levels. At system level a qualitative model describes the system structurally and/or functionally over its interfaces at a high abstract level. The description is made with the aim of a behavioral simulation at system level. This allows a virtual check, whether the design meets the system specification.

The system partitioning can be carried out on the basis of physical processes, the number of connections between the subsystems, the expected activity of the compounds, the mathematical approaches to the description or the geometrical dimensions. By subdividing the total system in mechanical, optical, fluidic, acoustic and electric components, the overall 


\section{System}

Coupled component models und system environment,

\section{Function parameters}

\section{Component}

Coupled partial models of the components,

Function parameters

\section{(Function-) Element}

2D/3D partial DE-Systems of the element,

Geometry and material parameters

\section{Process/ Technology}

2D/3D partial DE-Systems of the manufacturing processes,

Process parameters

\section{Example}

Vibrating tuning fork gyro sensor with piezomagnetic excitation and sensing

Magnetic network model of the tuning fork including drive coil influence

Magnetic Finite-Element-mode of the permanent magnet field setting the magnetic bias in the sensing arm
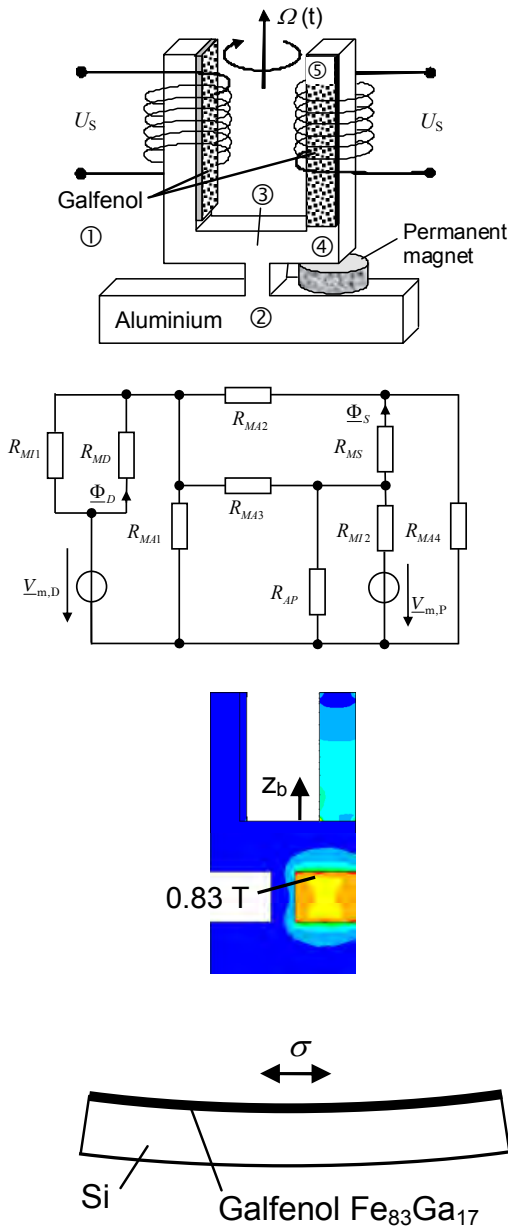

Fig. 1. Model hierarchy of complex systems and of a vibrating tuning fork gyro sensor with piezomagnetic excitation and sensing.

system are described clearly. For a component, several descriptions exist. They result e.g. from various design alternatives. This way, the complexity of a system description can be varied. These are simple models for a rough design, fast models for optimization calculations and highly exact models for the verification of a selected design variant. The different expenditures of the individual models results here not only from the expenditure for the computation of a model but also from the expenditure for the setup of the model. In particular, the expenditure to create completely new models is often higher than the computational expenditure.

The element plane includes function and form elements such as resistors or bending beams. Several elements form a system component. Typically items are described in two and three dimensions with partial differential equations (PDEs) and boundary conditions, which include geometric and material parameters. Accordingly, a high computation and simulation effort results. The expense is reduced if only the terminal behavior of the component is considered in form of a network description. Spatial de- pendencies are removed by employing ordinary differential equations (ODEs) as the PDE solutions. It is one objective of the Combined Simulation to determine parameters of network models from the 2D and 3D FE-models.

An element may be created on process and technology level virtually applying a process or technology simulator. The individual production processes are controlled by process parameters, which affect the geometry and material parameters of microsystem elements.

In Fig. 1 several partial models of a vibrating tuning fork gyro sensor are assigned to these levels of hierarchy [6]. One piezomagnetic plate is placed on each arm but perpendicular to each other. Both plates are surrounded by a coil. One plate is used to generate a fork vibration by application of an AC magnetic field to this excitation arm. In case of a rotation about the yaw axis, Coriolis force causes an arm vibration perpendicular to the excited direction. The piezomagnetic material placed at the arm which is not directly excited, reacts with a change of its magnetic properties and causes a 
fluctuation of an adjusted bias magnetic field. Thus, a voltage is induced in the surrounding detection coil which shows spectral components proportional to the yaw rotational rate. At process level the sputtering determines the mechanical bias of piezomagnetic layer and thereby its material parameters. The key process parameter is the process temperature.

The geometry parameters of the piezomagnetic unimorph arms define the properties of the piezomagnetic transducer. Assuming an ideally uniform magnetic field within the solenoid coils a behavioral description of the electromechanical system can be derived analytically in form of a network model [1]. This allows a behavioral simulation using a circuit simulator.

During a simulation an experiment is conducted on the models. The behavior of components and the system behavior are assessed on performance parameters which must lie within defined tolerance limits. Key performance parameters are, for example, the sensitivity of a sensor, its power consumption, temperature range, the output noise, but also its reliability, the extraction rate, and its cost $[7,8]$.

\section{Coupled System Simulation}

When a unified description with a single modeling language is not possible different component models can be coupled during system simulation as sketched in Fig. 2. The environment sets the boundary conditions. A coupling program controls the sequential automatic execution of various programs or types of analysis allowing iterative optimization calculations. This procedure is generally known as Co-Coupled Simulation or Simulator Coupling. Basics were described by Schwarz [9] and a coupling program presented by Schneider et al. [10].

The presented work concentrates on either network methods, FE-methods or the connection of both methods to derive custom-made models. Besides calculating with the ODEs instead of PDEs network methods benefit from concentration of the object properties in fewer elements. By its efficiency and performance network methods are prevalent for forecasting electromechanical systems behavior.

For some design tasks, however, the prediction only with network methods reaches its limits, when, for example, the degree of approximation of the network model is too low or the creation of the network model requires an excessive effort. In such cases a combination of network methods with another simulation method may be advantageous. It has been shown in particular that the combination with FE methods or

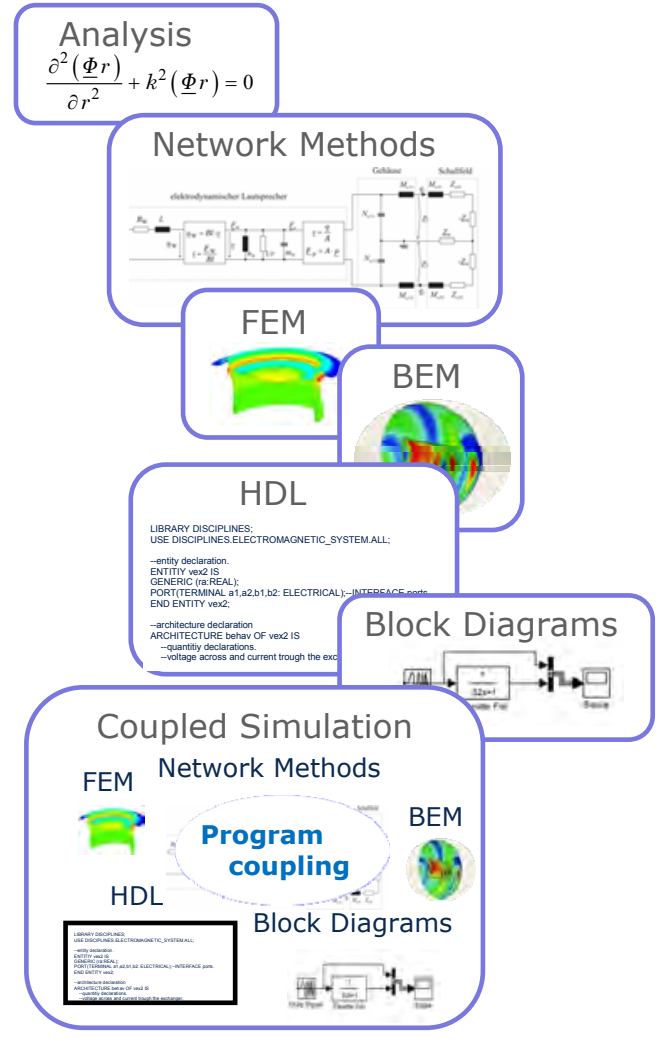

Fig. 2. Coupling of different programs or types of analysis

with BE-methods is a positive complement. FEmethods are to prefer when complex geometries \& continua are present. FE methods can handle material nonlinearities and limited model areas. Unlimited model areas are covered by far-field elements.

An important aspect of simulator coupling is the software dependence. A new version of a simulation program can endanger the proper functioning of the coupled simulation. This disadvantage does not apply if a software developer itself couples various simulation programs. An example is the integration of the Simplorer soft-

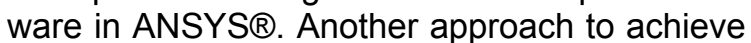
software independence is the Combined Simulation. Hereafter, the term is introduced for general methods of network interconnection with a further simulation method.

\section{Combined System Simulation}

In contrast to Coupled Simulation the Combined Simulation links several simulation methods at user level. The user generates virtual objects using one method, which he installs in the user interface of another different method. In the data fields are not intervened thereby, i.e. the user does not create coupling programs.

With the combination of network methods with FE methods, the performance capabilities of the 


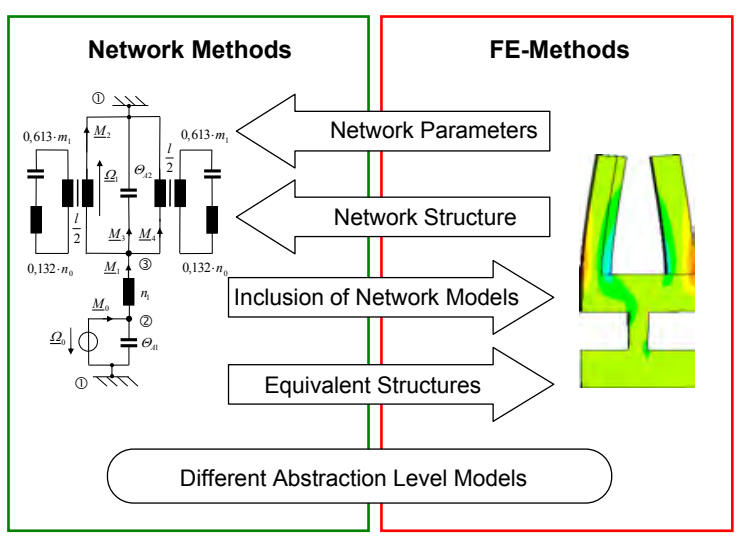

Fig. 3. Applications of Combined Simulation.

two methods are complementary advantageous. The use of problem-adapted models, which are reduced to a concrete question allows a fast optimization of the design. Feedback between simulators is not provided and can therefore not call sequential timeconsuming FE simulations.

Between five application fields of Combined Simulation can be distinguished (see Fig. 3):

1. use of FE models to determine the parameters of components of network representations,

2. use of FE models to determine the structure of network representations,

3. integration of network models in an FE model,

4. application of network methods to the creation of equivalent structures for the efficient calculation of a FE model,

5. modeling of the problem at different abstraction levels.

Combined Simulation is a powerful simulation method that takes into account the available and manageable tools and methods of a typical $R \& D$ engineer. The methodology promotes the understanding of the operation of a system. This effect is essential for the design of powerful products. Nevertheless, no commercial tool which incorporates all applications of Combined Simulation is available today.

Various applications of the Combined Simulation are extensively studied in [5]. In the next sections the different applications of Combined Simulation are explained by hand of examples.

\section{Determining Network Parameters}

Unknown network parameters can be computed often by simple FE-simulations, e.g. when complex geometries \& continua are present. The network coordinates of an element follow from the FE-analysis results in a direct approach.

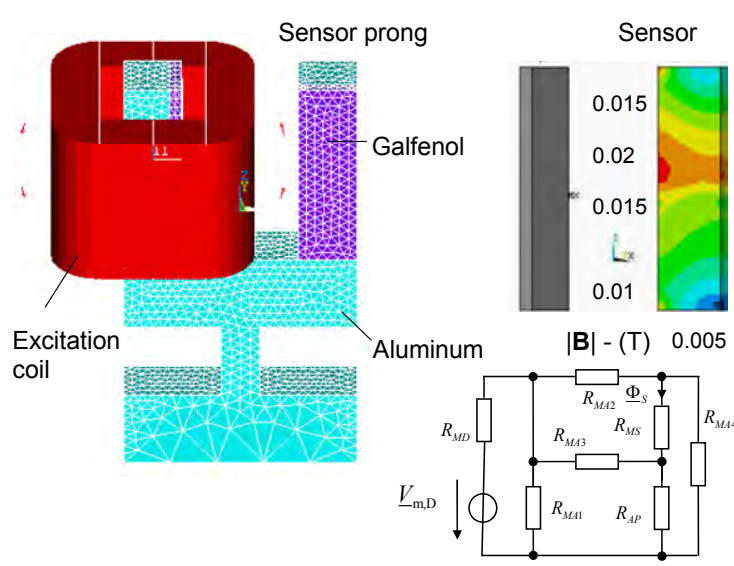

Fig. 4. Drive coil field simulation

From energy-storing elements alternatively only one network coordinate and the related energy is needed. When the network coordinates are difficult to access or network elements of a structure are not physically existent, then also the model of a larger system, which includes the elements of interest, can be used to determine the searched parameter.

An example for the determination of network parameters with FE simulations is the magnetic circuit representation of the tuning fork gyro in Fig. 1. Sensor coil and drive coil form an undesired loose coupled transformer, i.e. the drive coil magnetic field influences the voltage induced in the detection coil. The magnetic flux in the sensor plate produced by an alternating 100 $\mathrm{mA}$ current in the 300 turns drive coil, is depicted in Fig. 4. The magnetic flux density reaches $1 / 20$ of the flux density caused by the permanent magnet at the maximum.

To simplify the magnetic network the influence on the magnetic operating point and the distribution of this magnetic field contribution is not considered.

\section{Determining Network Structures}

The obtainable accuracy of a network model is low compared to FEM, especially for complex structures, but sufficient in most cases.

When non-linear behavior of a system can be neglected and linear behavior assumed, a network structure can be determined by applying linear network theory. Following this theory an $\mathrm{N}$-port-matrix establishes the relations between $\mathrm{N}$-port interfaces of a multi-physics system. The unknown elements of the N-port can be computed with the help of simple FE-analysis. In the last step the $\mathrm{N}$-port is transferred into a circuit which is constructed of basic network elements.

In Fig. 5 the procedure is applied to an electroacoustic transducer, e.g. a piezoelectrical actuator. The transducer can be described with a lossless transformer-like coupling 2-port: 

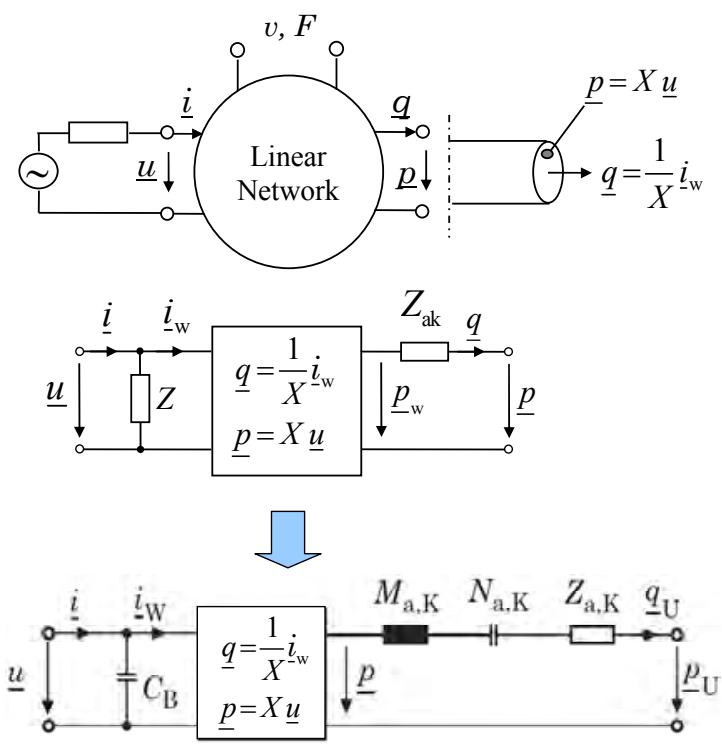

Fig. 5. Determination of 2-port representation parameters of an electroacoustic transducer

$$
\begin{aligned}
& \left(\begin{array}{l}
u \\
i
\end{array}\right)=\left(\begin{array}{ll}
\alpha_{11} & \alpha_{12} \\
\alpha_{21} & \alpha_{22}
\end{array}\right)\left(\begin{array}{l}
p \\
q
\end{array}\right) \\
& \alpha_{11}=\frac{1}{X}, \alpha_{12}=\frac{Z_{a k}}{X}, \\
& \alpha_{21}=\frac{X}{Z}, \alpha_{22}=\frac{Z_{a k}}{Z X}+X
\end{aligned}
$$

The transduction coefficient $X$ is real while the electrical impedance $Z$ and acoustic impedance $Z_{\text {ak }}$ are complex. This representation is particularly appropriate when $X$ is frequencyindependent. The network parameters can be determined starting with several FE-analyses, where the boundary conditions are varied. From the obtained transfer functions the impedances can be further expressed by network elements.

\section{Inclusion of Network Structures in FE- Models}

As mentioned, often systems can be modeled more efficient or precise with FE-methods. Nevertheless, if a transducer principle is not supported by the FE-program or if the setup of a FE-model of the subsystem requires an inappropriately high expenditure, it can be appropriate to describe subsystems with network models within the FE-model. For many transducers all parameters of the network model are given by the manufacturer while material parameters are proprietary information.

Suitable interfaces between FE-model and network model are planes of sections within ranges with approximately constant field quantities and homogeneous field distribution. Individual finite elements offered by the respective
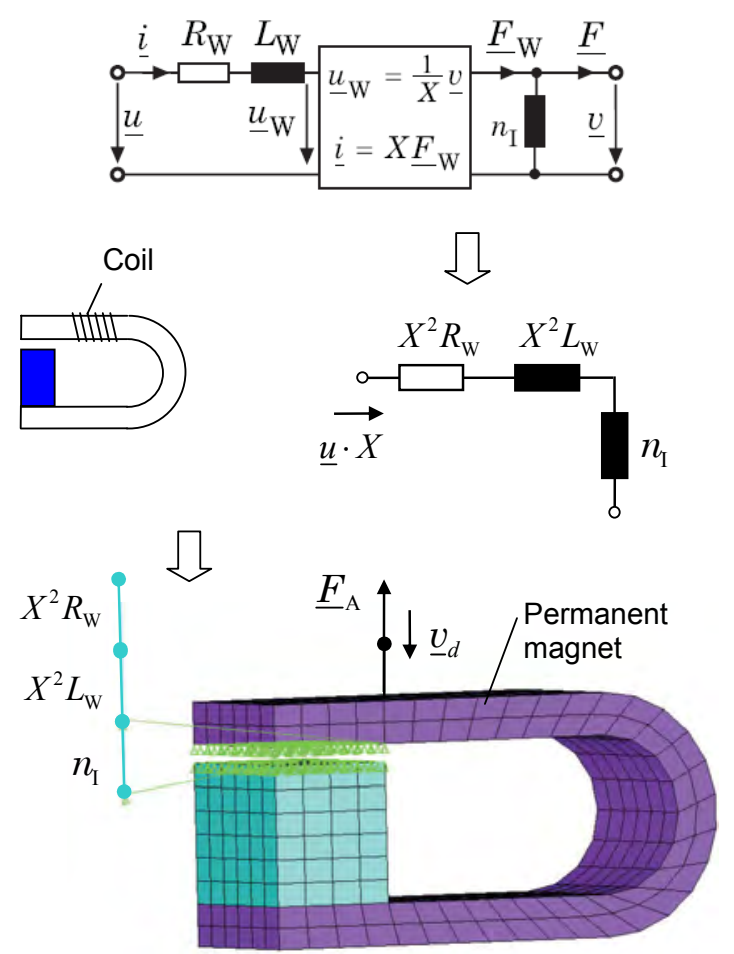

Fig. 6. Inclusion of an electromagnetic actuator network model in an FE-model of a permanent magnet

FE-program are used to represent discrete elements. The overall system behavior is computed exclusively by the FE-program.

Fig. 6 shows the computation of an electromagnetic actuator as an example [5]. The three-dimensional FE-modeling of the coil would lead here to an inadequately high expenditure. The well-known electromechanical network model of a solenoid coil is a sufficient approximation in the frequency range to be modeled. All parameters are given by the transducer manufacturer for the network model. Before inclusion in the FE-model the electrical elements are transformed to the mechanical side. Then the transducer can be removed. The resulting network representation is included by special finite elements in the FE-model and coupled with the FE-model of the magnet. The dynamic behavior is simulated with the FEprogram via transient or harmonic analysis.

\section{Creation of Equivalent Network Structures}

Contrary to the approach presented in the previous section the goal here is to find an efficiently describable equivalent structure for a subsystem, which enables a closed computation of a FE-model. The equivalent structure can be a model of another physical connection or exhibit another form and other geometrical dimensions. It models only the effect of the original system in an operational range with sufficient approximation degree. The need 
might arise when the construction with elementary network units is not possible. Network methods are used here in order to find the equivalent structure. The method does not lead compellingly to a solution.

The method was applied to the description of friction losses in a hip joint [11]. Fig. 7 visualizes the bearing of the femur head in the acetabulum. The wedge-shaped gap cannot be properly modeled with finite elements. Alternatively the linear viscous friction part can be modeled by translational friction elements which are connected to the femur head by ideal rods as shown in Fig. 8.

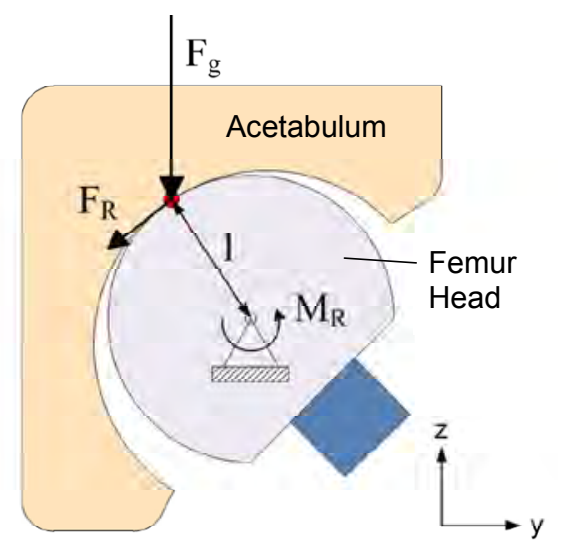

Fig. 7. Hip joint

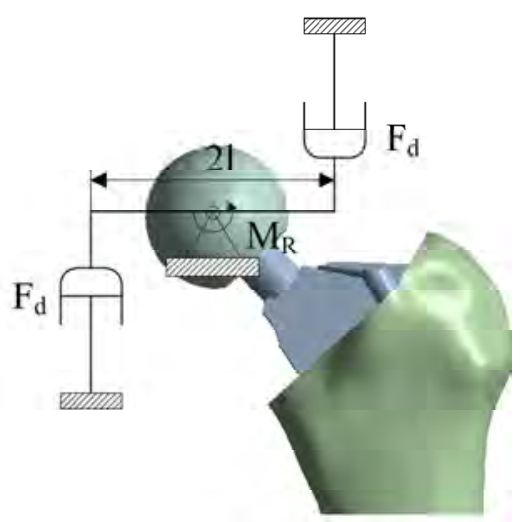

Fig. 8. Equivalent structure of the friction

\section{Outlook}

Combined Simulation is a powerful and flexible applicable method which is often favorable for the behavioral simulation of electromechanical systems instead of using only a single modeling and simulation method. Combined Simulation offers a good compromise between managability, expenditure for the model setup, computing time and accuracy.

Since a fully automated predetermination of systems behavior is already possible and desired, at the same time the understanding of the system operation is a basic prerequisite for an optimal product design. With Combined Simula- tion models at different abstraction levels can be created and different ways of thinking applied to solve a problem. Thus the simulation process can serve as a source of new ideas.

The integration of network analysis programs in widespread system simulation programs during the last years demonstrates that the capabilities and efficiency of this method combination was recognized. Although the simulation tool developer ensures the compatibility of coupling programs in this new tools, a pure coupling of two simulation methods is much less powerful than an adaption of the methods to a problem depending on the respective task by the user.

\section{References}

[1] Lenk, A., Ballas, R.G., Werthschützky, R., Pfeifer, G.: Electromechanical Systems in Microtechnology and Mechatronics, Springer, (2010).

[2] Janschek, K., Systementwurf mechatronischer Systeme (in German), Springer, Berlin Heideberg; 1. Auflage, (2010).

[3] Schneider, P.: Modellierungsmethodik für heterogene Systeme der Mikrosystemtechnik und Mechatronik, TUDpress Verlag der Wissenschaften, Dresden, (2010).

[4] Eccardt, P.-C., Knoth, M., Ebest, G., Landes, Clauß, C., and Wünsche, S.: Coupled finite element and network simulation for microsystem components. In Proc. MICRO SYSTEM Technologies '96, pp. 145-150, VDE-Verlag, 1996.

[5] Starke, E.: Kombinierte Simulation - eine weitere Methode zur Optimierung elektromechanischer Systeme, TUDpress Verlag der Wissenschaften, Dresden, 2010.

[6] Marschner, U., Yoo, J.-H., Starke, E., Graham, F., Mudivarthi, C., Fischer, W.-J. and Flatau, A.B.: Electromechanical network modeling applied to magnetoelastic gyro sensor design. Proc. SPIE, Vol. 7647, 76472S (2010); doi:10.1117/12.847782

[7] Gerlach, G.; Dötzel, W.: Introduction to Microsystem Technology. John Wiley \& Sons, 2008

[8] Physics of Semiconductor Sensors. Kapitel in: Trah, H.-P.; Franz, J.; Marek, J.: Advances in Solid State Physics. Vieweg, Braunschweig, Wiesbaden, 1999, S. 25-36

[9] Schwarz, P.: Systemsimulation. In: Fischer, W.-J. (Ed.): Mikrosystemtechnik, pp. 351-374. Vogel, Würzburg, 2000.

[10] Schneider, P., Schneider, A., Bastian, J., Reitz, S., and Schwarz, P.: Moscito - a program system for mems optimization. In Proc. DTIP Conference, Cannes, May 5-8 2002.

[11] Körbitz, R.: Modellierung und Simulation des dynamischen Verhaltens einer sensorischen Hüftprothese im Knochenverbund unter Berücksichtigung besonderer Randbedingungen, Diploma th., IHM, Technische Universität Dresden, 2011 